\title{
Experimentación, comportamiento y modelación de la tapia pisada
}

\author{
A rammed earth experimentation, behavior and modelling
}

Fecha de Recepción: 12 de octubre de 2013

Fecha de Aprobación: 09 de noviembre de 2013

\author{
Nelson Afanador García* \\ Mayerly Carrascal Delgado** \\ Marvin Joseph Bayona Chinchilla***
}

\begin{abstract}
Resumen
Las edificaciones consideradas patrimonio histórico de la nación son construcciones en tierra apisonada, levantadas por artesanos que ven en la tierra un material ambientalmente aconsejable para el uso en construcción, pero sin ningún análisis técnico de las propiedades físicomecánicas de la tapia pisada y de las solicitaciones a que se ve expuesta.
\end{abstract}

Este artículo presenta los principales resultados de una investigación que buscó determinar las propiedades físico-mecánicas y el comportamiento de los elementos estructurales que constituyen una construcción en tapia pisada, con los cuales se verificó y validó un modelo matemático para determinar el grado de vulnerabilidad del Complejo Histórico Gran Convención (Ocaña, Norte de Santander), usando técnicas de modelación en elementos finitos.

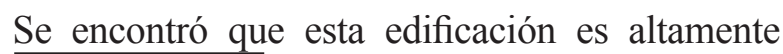

\begin{abstract}
The buildings considered a historical heritage of the nation are rammed earth constructions, built by craftsmen who see earth as an advisable and environmentally material feasible for construction, but without any physical and mechanical properties' technical analysis of the rammed earth and the stresses to which they will be exposed.
\end{abstract}

This article summarizes the investigation main results, to determine the physical and mechanical properties and the behavior of the elements constituting structures on a rammed earth construction, with which it was verified and validated a mathematical model, to determine the vulnerability degree that would be subject the Great Convention Historical Complex, by using modeling techniques in finite elements. It was found that this building is highly vulnerable to different events.

\footnotetext{
* M.Sc. Universidad Francisco de Paula Santander, Sección Ocaña (Colombia). nafanadorg@ufpso.edu.co

** Universidad Francisco de Paula Santander, Sección Ocaña (Colombia).

*** Universidad Francisco de Paula Santander, Sección Ocaña (Colombia).
} 
vulnerable a diferentes acciones. Mediante técnicas de cinética de secado se determinó el tiempo de fraguado de diferentes tamaños de especímenes, con el fin de establecer el tiempo de secado para muros realizados en tapia pisada con fines constructivos.

Palabras clave: Tapia pisada, Tierra apisonada, Resistencia tapia pisada, Cinética de secado.
The drying kinetics techniques allowed determining the different sizes specimens' setting time, in order to establish the drying time for rammed earth walls, made for construction purposes.

Keywords: Earth Walls, Rammed Earth, Rammed Earth Resistance, Drying Kinetics. 


\section{INTRODUCCIÓN}

Muchas construcciones en Colombia que datan de la época de la Colonia y que fueron construidas en tapia pisada (tierra apisonada), con material de la región y por artesanos locales, aún se mantiene en pie. Hace poco la tierra apisonada fue relegada, como la mampostería lo fue en los años 1950 a 1960 , cuando otros materiales alcanzaron su auge, principalmente el concreto en nuestro medio. Los materiales compuestos principalmente de tierra (tapia pisada) han basado sus diseños en experiencias pasadas sin ningún estudio científico y sin análisis previos a las edificaciones en condiciones inusuales o sujetas a cargas menos probables que las cargas gravitacionales o de uso. A pesar de que en Colombia la construcción en tapia pisada no es permitida, en sitios particulares como el Occidente de Australia [1, 2], Canadá y Estados Unidos (California) [3] es permitida, y además ha sido evidente el interés de Inglaterra en este tipo de construcciones, pues allí han publicado recientemente una guía de construcción [4], acompañada de diseños arquitectónicos muy interesantes.

A la fecha, la tapia pisada ha sido relegada como material estructural, y pocos estudios se han desarrollado con el fin de permitir su uso, al menos en zonas de poca amenaza sísmica y con pocas precipitaciones atmosféricas, para dar soluciones integrales de bajo impacto ambiental y rescatar los rasgos culturales de la comunidad. En zonas de importante actividad sísmica, la alta vulnerabilidad de estos monumentos históricos ha sido claramente expuesta en muchos sitos y en diferentes escenarios, pues ante terremotos moderados ha provocado colapso y la pérdida de vidas humanas debido a su comportamiento sísmico. Es por esto que es importante desde la academia participar en investigaciones tendientes a definir el comportamiento del material y evaluar la vulnerabilidad sísmica a que están expuestas iglesias, auditorios y viviendas de la época de la Colonia, con el propósito de disminuir el peligro al que podrían estar expuestas estas construcciones; esto ha sido una preocupación de la línea de investigación en materiales y sísmica de la Universidad Francisco de Paula Santander Ocaña (UFPSO).

\section{Metodología}

La materia prima utilizada corresponde al municipio de Ocaña, Norte de Santander, enmarcado en las coordenadas geográficas $X=1.077 .500$ a 1.082 .000 y $\mathrm{Y}=1.401 .000$ a 1.407 .000 , con una extensión aproximada de $7.74 \mathrm{~km}^{2}$ de área urbana [5], donde hay una presencia importante de casas, iglesias, conventos, bibliotecas y diferentes entidades construidas en tapia pisada, algunas levantadas entre los años 1950 y 1960 [6], pero otras, como iglesias, conventos y el complejo histórico de la Gran Convención, construidas entre los años 1533 y 1583 [7], las cuales aún se encuentran en pie, pero con algunas patologías debido a la acción del viento, a eventos sísmicos y a cambios climáticos. El tipo de suelo utilizado en la construcción de muros en tapia pisada fue tomado de construcciones ubicadas en diferentes sitios, como se indica en la Fig. 1, con el fin de caracterizar las muestras de suelo y determinar si el tipo de suelo de la muestra de trabajo es similar al utilizado en este tipo de edificación, mediante la clasificación de suelos, porcentaje de finos y gruesos, límites de Attemberg, granulometría por tamizado en seco y por sedimentación (hidrómetro) y ensayo de compactación proctor para determinar la humedad óptima.

Las propiedades mecánicas de la tapia pisada fueron determinadas mediante la construcción de especímenes de 250x500x500 mm, con el fin de determinar la resistencia última a compresión, el módulo de elasticidad longitudinal, el módulo de rotura (paralelo y perpendicular a la junta), la resistencia a la tracción, el módulo de corte y la relación de Poisson. 


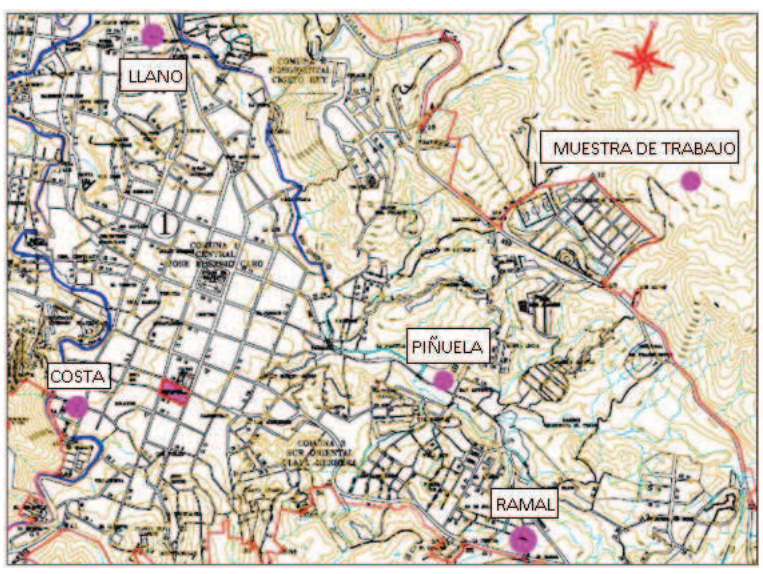

Fig. 1. Ubicación de las muestras de suelo y la muestra de trabajo [5]

El equipo utilizado en los ensayos fue una máquina universal de ensayos sistematizados PU-100, calibradores digitales, gatos hidráulicos con celdas de carga hasta $100 \mathrm{KN}$, comparadores de carátula con salida RS232 y dispositivos electrónicos LVDT.

El ensayo a compresión normal utilizó dos comparadores de carátula para medir los desplazamientos y evitar la distorsión en los datos [8], como se ilustra en la Fig. 2, y determinar las curvas esfuerzo-deformación de los especímenes y el módulo de elasticidad longitudinal bajo el criterio de la línea secante correspondiente a la 50 millonésima de la deformación y $40 \%$ del esfuerzo máximo [9]. El módulo a la rotura fue determinado para las condiciones de carga paralela y perpendicular a la junta de construcción mediante la aplicación de carga en el centro de la luz y una condición de tres apoyos [10] (ver Fig. $3)$.

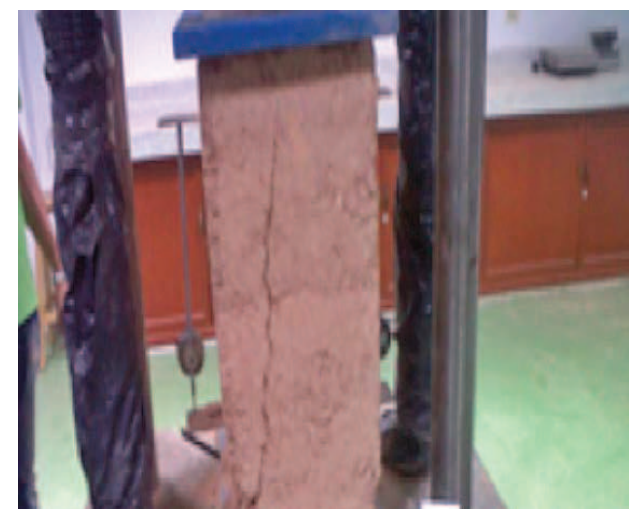

Fig. 2. Ensayo a compresión normal [6] (Tomado Lab. Resistencia de materiales y sísmica UFPSO)

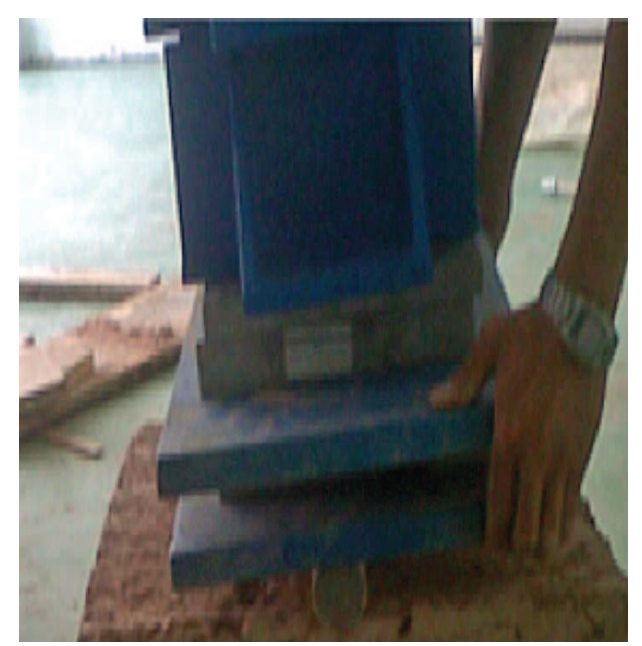

Fig. 3. Ensayo Módulo de rotura paralela a la junta. [6] (Tomado Lab. Resistencia de materiales y sísmica UFPSO)

La resistencia al corte, el módulo de elasticidad al corte y la relación de Poisson fueron determinados [11] utilizando LVDT con precisión de 1 micra, para medir la extensión y el acortamiento del murete de 250x500x500 mm; la Fig. 4 ilustra el proceso. 


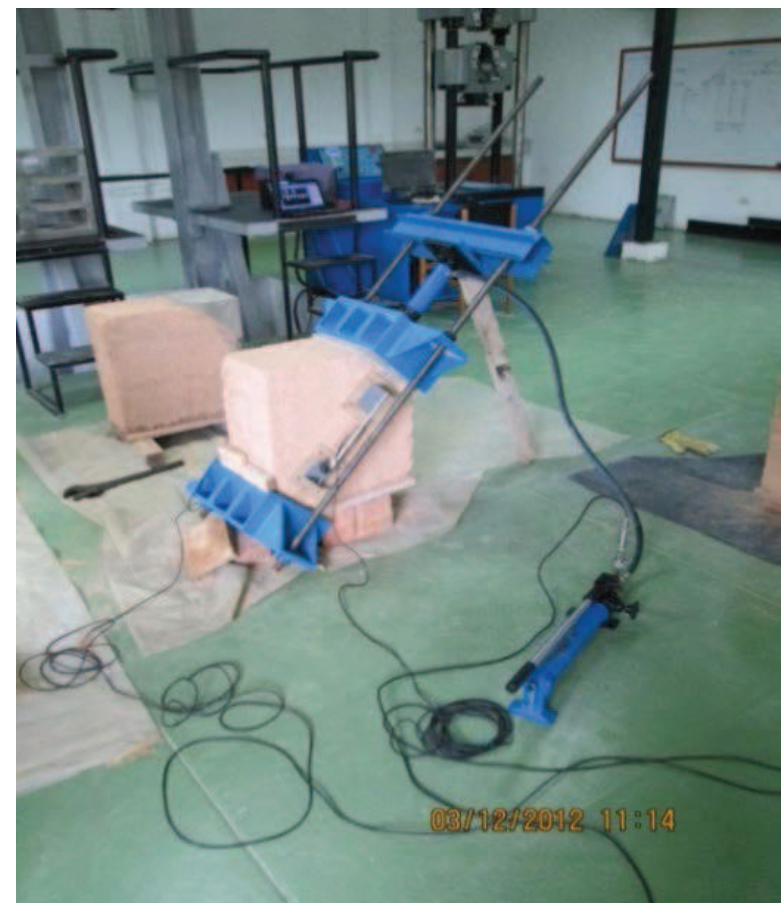

Fig. 4. Ensayo a Tracción en Muretes de Tapia Pisada [6] (Tomado Lab. Resistencia de materiales y sísmica UFPSO)

Adicionalmente se realizó una cinética de secado, con el fin de determinar la pérdida de peso con el tiempo y establecer tendencias de secado; además, fue hallada la variación de la resistencia a la compresión en función del tiempo. Se determinó una muestra representativa mediante un muestreo aleatorio simple, cuyo tamaño fue de 43 especímenes con las siguientes

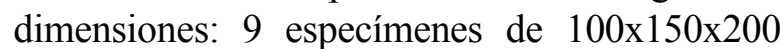
$\mathrm{mm}, 9$ de 150x150x200 mm y 9 de 200x150x200 $\mathrm{mm}$; estos especímenes fueron elaborados en tres capas compactadas de $50 \mathrm{~mm}$ cada una con pisón de madera de $2.4 \mathrm{~kg}$ y 100 golpes por capa; adicionalmente, se elaboraron 16 especímenes de 250x500x500 mm, construidos en 7 capas compactadas a $70 \mathrm{~mm}$ con pisón de madera de 4 y $3 \mathrm{~kg}$ (el pisón de $3 \mathrm{~kg}$ tiene forma cónica para uso en las esquinas).

\section{Resultados}

La distribución de partículas de tamaño grueso se puede observar en la Fig. 5, donde la muestra denominada El Ramal tiene mayor porcentaje de finos que las otras muestras, según el porcentaje que pasa el tamiz 200, mientras que la muestra de trabajo se encuentra en el rango de muestras tomadas al azar para determinar las características de los materiales.

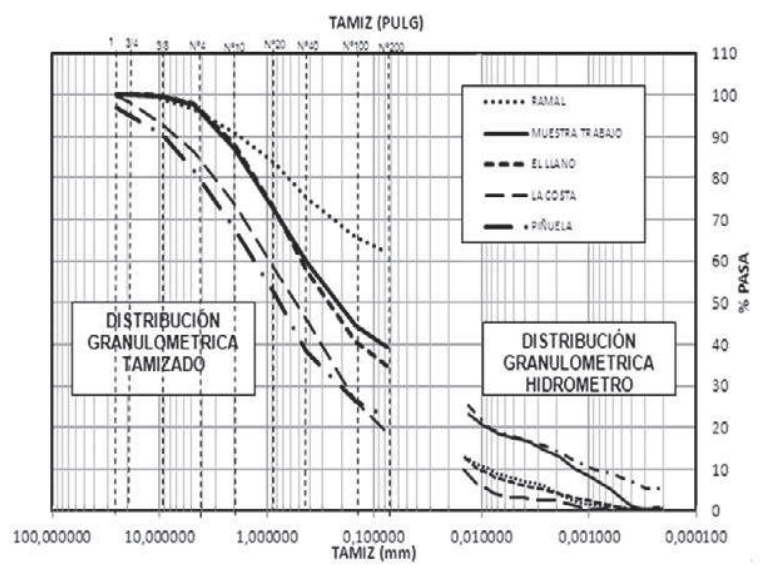

Fig. 5. Distribución granulométrica por tamizado en seco y por hidrómetro.

En la Tabla 1 se indica la clasificación del suelo (según sistema unificado de clasificación de suelos), realizada a una distribución de tamaño de partículas por tamizado en seco [12] y por hidrómetro [13] para las diferentes muestras de suelos y para la muestra de trabajo utilizada en la elaboración de la tapia pisada. La densidad de las muestras oscilan entre 1.72 a $1.99 \mathrm{gr} / \mathrm{cm}^{3}$, con una clasificación de suelos que se encuentran entre arenas limo-arcillosas y arenas arcillosas hasta arcillas de baja plasticidad, donde la muestra de trabajo tiene una clasificación de arena limoarcillosa con porcentajes de finos del $59.9 \%$ y de gruesos $40.1 \%$, la cual es representativa del material utilizado en las construcciones en tapia pisada. 
TABLA 1

Clasificación DE SUElos

\begin{tabular}{|c|c|c|c|c|c|c|c|c|}
\hline $\begin{array}{l}\text { Lugar de } \\
\text { Muestreo }\end{array}$ & \begin{tabular}{|l}
$\begin{array}{c}\text { Densidad } \\
\text { (gr/cm3) }\end{array}$ \\
\end{tabular} & LL\% & LP\% & IP\% & & Clasificación & \begin{tabular}{c|}
$\%$ \\
Gruesos \\
\end{tabular} & $\begin{array}{c}\% \\
\text { Finos }\end{array}$ \\
\hline E1 llano & 1.72 & 26.69 & 20.98 & 5.70 & SC-SM & Arena Limo - Arcillosa & $42.34 \%$ & $57.66 \%$ \\
\hline La piñuela & 1.88 & 36.36 & 25.42 & 10.93 & $\mathrm{SC}$ & Arena Arcillosa & $61.70 \%$ & $38.30 \%$ \\
\hline El ramal & 1.96 & 33.28 & 17.95 & 15.32 & $\mathrm{CL}$ & Arcilla de Baja Plasticidad & $24.79 \%$ & $75.21 \%$ \\
\hline $\mathrm{La}$ & & & & 5.60 & 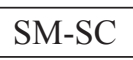 & & $54.16 \%$ & $45.84 \%$ \\
\hline $\begin{array}{c}\text { Muestra de } \\
\text { Trab. }\end{array}$ & 99 & 33.99 & 26.74 & 7.25 & SM-SC & Arena Limo - Arcillosa & $40.07 \%$ & $59.93 \%$ \\
\hline
\end{tabular}

Los límites de Attemberg, consistentes en el límite plástico e índice de plasticidad [14] y límite líquido [15], indicados en la Tabla 1, permiten establecer que son muestras de baja plasticidad, pues los límites líquidos son inferiores al 50\%. La humedad óptima obtenida [16] para la muestra de trabajo fue de 13,2\%; para una densidad máxima de $1,985 \mathrm{gr} / \mathrm{cm}^{3}$ de los especímenes utilizados el contenido de humedad fue de $11,17 \%$, cercano al óptimo [6].

El comportamiento a la compresión de la tapa pisada es lineal, con muy poco o ninguna incursión en el rango no lineal, como se puede observar en la Fig. 6, donde los especímenes tienen un comportamiento elástico lineal, mientras que 2 de los 6 especímenes realizaron alguna incursión en el rango no lineal. El esfuerzo a la compresión promedio a los 28 días fue de $0.17 \mathrm{MPa}$, con módulo de elasticidad (E) máximo de $74.00 \mathrm{MPa}$, mínimo de $42.12 \mathrm{MPa}$ y $\mathrm{E}_{\text {promedio }}$ de $54.78 \mathrm{MPa}$ [6].

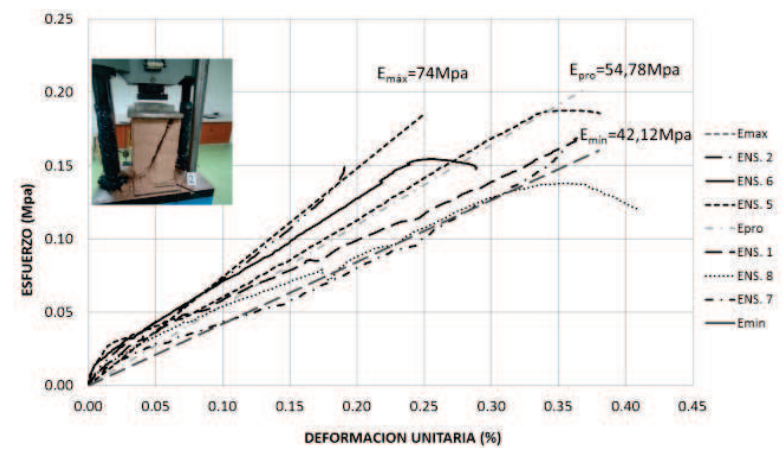

Fig. 6. Comportamiento a compresión y módulo de elasticidad estático de la tapia pisada [6]
El módulo de rotura fue determinado a 4 especímenes de 250x500x500 mm en la dirección perpendicular a la junta, cuya carga máxima fue de 2,103 KN y Módulo de rotura promedio de $0,042 \mathrm{MPa}$ [6].

El comportamiento de la tapia pisada a tracción diagonal no está definido, por el contrario, depende de la compactación, del contenido de humedad y de la manipulación a que está sujeta, aunque en la mayoría de los casos se define un rango lineal, para que posteriormente se presenten grandes deformaciones con poco o ningún aumento del esfuerzo cortante (véase la Fig. 7).

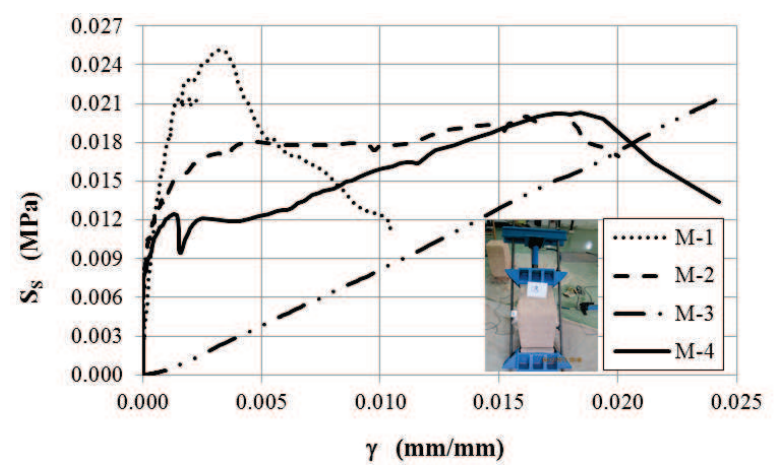

Fig. 7. Curvas esfuerzo cortante vs. deformación angular [6]

La resistencia máxima a corte promedio para la tapia pisada ensayada fue de $0.022 \mathrm{MPa}(22$ $\mathrm{KPa}$ ), con un coeficiente de variación de $9.9 \%$ y un módulo de corte promedio de $0.989 \mathrm{MPa}$ $(989 \mathrm{KPa})$, con un coeficiente de variación del $35.8 \%$ (véase Tabla 2). Con los datos obtenidos se estableció la relación de Poisson n, de 0.26. 


\section{TABLA 2}

RESisTenCia MÁXima y Módulo DE CORTE, ENSAYO DE TRACCIÓN DIAGONAL EN TAPIA PISADA [6]

\begin{tabular}{|c|c|c|}
\hline Espécimen & Ss ÍMoa) & G (Moa) \\
\hline M-1 & 0.025 & 1.586 \\
\hline $\mathrm{M}-2$ & 0.020 & 0.877 \\
\hline M-3 & 0.021 & 0.661 \\
\hline IVM & 0.020 & 0.830 \\
\hline Promedio & 0.022 & 0.989 \\
\hline Desvest.(Mpa) & 0.002 & 0.354 \\
\hline Coef Var. (\%) & 9.9 & 35.8 \\
\hline
\end{tabular}

\section{CAso de estudio}

El Complejo Histórico de la Gran Convención (CHGC), patrimonio histórico y cultural de la nación, fue construido por la comunidad Franciscana hacia los años 1533 y terminado bajo la ermita del convento de San Antonio hacia 1583 [7]. Esta construcción ha tenido diversos usos, desde convento, escuela, colegio, cuartel y universidad [17]. El CHGC ha soportado sismos de diferente intensidad y magnitud, por ejemplo, el 28 de abril de 1894 el frontis de la iglesia se deterioró gravemente como consecuencia del terremoto de Cúcuta, por lo que su espadaña original fue reemplazada por la torre que hoy ostenta [18] (ver Fig. 8).

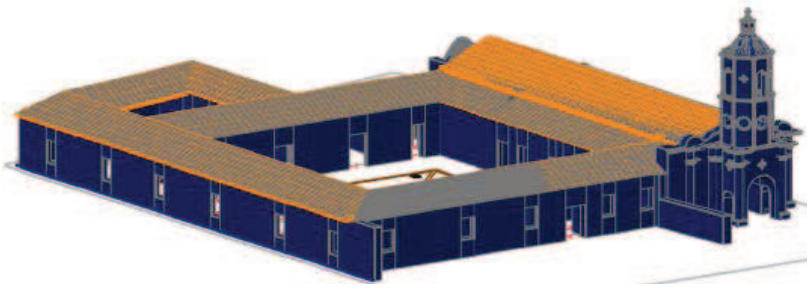

Fig. 8. Complejo histórico de la Gran Convención en 3D [6]

El periodo aproximado del CHGC fue calibrado usando los últimos dos reglamentos colombianos de construcción, así:
Dirección X-X de $0.028 \mathrm{~s}$

Dirección Y-Y de 0.027 s [19]

Dirección X-X de $0.022 \mathrm{~s}$

Dirección Y- Y de 0.020 s [20]

Para efectos del cálculo de la fuerza sísmica en la base es indiferente el periodo aproximado Ta (seg), pues estos se encuentran en la meseta del espectro elástico de diseño donde la aceleración espectral es la misma para todos los periodos hallados. El peso de los muros es de 16.655,87 $\mathrm{KN}$, la cubierta en teja de barro y madera rolliza es de $826,39 \mathrm{KN}$, para un peso total de $17.482,26$ $\mathrm{KN}$ y un cortante sísmico en la base de $8.741,13$ $\mathrm{KN}$.

La modelación numérica fue realizada en SAP 2000 Ver 14 [21], con muros discretizados en elementos finitos rectangulares de 250x250 mm, con 37 y 36 muros en la dirección X-X y Y-Y, respectivamente, con espesores que varían desde $390 \mathrm{~mm}$ hasta $1070 \mathrm{~mm}$. El análisis estático lineal fue realizado mediante una simulación por elementos finitos, aplicando fuerzas en los elementos superiores de la malla en proporción al porcentaje de participación de la rigidez de cada muro en la dirección de análisis y donde los efectos sobre el suelo fueron tenidos en cuenta mediante la colocación de elementos tipo Spring (joint spring) con rigidez en las direcciones locales 1,2 y 3 , dadas en función de la constante de balastro $\left(\mathrm{K}=25.000 \mathrm{KN} / \mathrm{m}^{3}\right)$ y f.

La modelación fue alimentada con los resultados de laboratorio realizados, y sus resultados son indicados en la Fig. 9, donde el índice de flexibilidad fue de 3.1 y 3.6 en la dirección X y $\mathrm{Y}$, respectivamente; un índice muy alto para una zona con amenaza sísmica intermedia, ya que la aceleración pico efectiva $\left(\mathrm{A}_{\mathrm{a}}\right)$ es de $0.2 \mathrm{~g}$. 


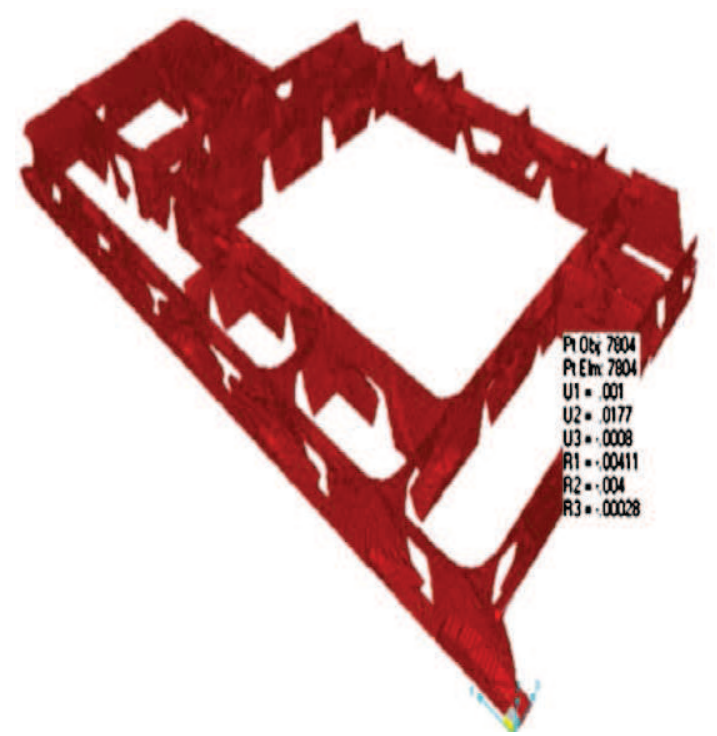

Fig. 9. Modelación en elementos finitos, en SAP 2000 [6]

Debido a que la tapia pisada solo tiene resistencia a la compresión y, en menor medida, a la flexión, se determinaron los índices de sobreesfuerzo a compresión y flexión. La modelación para determinar el índice de sobrerresistencia a la compresión se ilustra en la Fig. 10, para la condición envolvente (carga muerta y sismo), y esfuerzos S22.

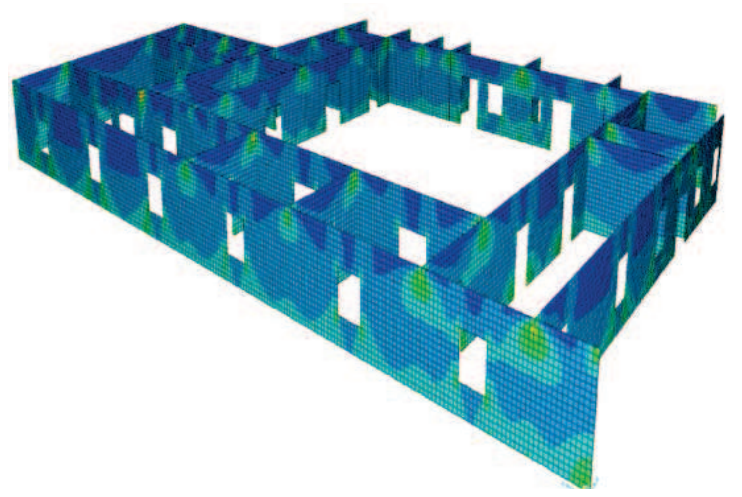

Fig. 10. Modelación en elementos finitos índice de sobre resistencia a compresión [6]

El índice de sobreesfuerzo a compresión ( $\left.\mathrm{IS}_{\mathrm{C}}\right)$, determinado mediante la expresión (1), tuvo en cuenta los problemas de eflorescencias y pequeños desprendimientos de pañete, como también una reducción de la resistencia a la compresión.

$$
I S_{C}=\frac{S S_{22}}{\phi * \varphi_{C} * \varphi_{D} * \sigma_{C}}(1)
$$

$\mathrm{f}=$ Coeficiente de reducción de resistencia a compresión, 0.8

$\mathrm{j}_{\mathrm{C}}=$ Coeficiente de reducción por conservación, 0.9

$\mathrm{j}_{\mathrm{D}}=$ Coeficiente de reducción por diseño, 1.0

$\mathrm{s}_{\mathrm{C}}=$ Resistencia a la compresión mediante ensayos 
Los $\mathrm{IS}_{\mathrm{C}}$ son muy similares en las dos direcciones, estánalrededorde2.6,yelÍndice de Vulnerabilidad por sobreesfuerzo a compresión (carga muerta y sismo) es de 0.38 (véase Tabla 3).

\section{TABLA 3}

ÍNDICE DE SOBRERRESISTENCIA E ÍNDICES DE VULNERABILIDAD A COMPRESIÓN POR ACCIONES GRAVITACIONALES Y SISMO [6]

\begin{tabular}{|c|c|c|c|c|}
\hline & $\begin{array}{c}\text { ISc } \\
(1,2 \mathrm{D}+1,6 \mathrm{Lr}) \\
\text { máx. }\end{array}$ & $\begin{array}{c}\text { ISc } \\
\text { (envolvente) } \\
\text { máx. }\end{array}$ & $\begin{array}{c}\text { índice de } \\
\text { Vulnerabilidad } \\
\text { por } \\
\text { sobre es fuerzo } \\
\text { a compresión } \\
(1,2 \mathrm{D}+1,6 \mathrm{Lr}) \\
\text { máx. }\end{array}$ & $\begin{array}{c}\text { índice de } \\
\text { Vulnerabilidad } \\
\text { por } \\
\text { sobre es fue rzo } \\
\text { a compresión por } \\
\text { envolvente máx. }\end{array}$ \\
\hline Dirección Y-Y & 0.47 & 2.52 & 2.12 & 0.39 \\
\hline Dirección X-X & 0.50 & 2.64 & 2.00 & 0.38 \\
\hline
\end{tabular}

El índice de sobreesfuerzo a flexión $\left(\mathrm{IS}_{\mathrm{F}}\right)$ fue hallado; aunque la resistencia a la flexión observada en laboratorio es pobre, su índice de vulnerabilidad, debido a la flexión, es igual a 0.017 (ver Fig. 11).

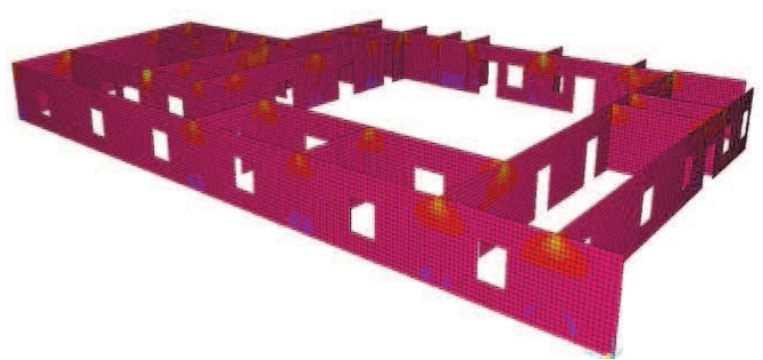

Fig. 11. Modelación en elementos finitos índice de vulnerabilidad a flexión [6]

Debido a que no se encuentran referencias que indiquen el fraguado de los muros en tapia pisada, en la Fig. 12 se elaboraron las curvas de peso normalizado promedio vs. hora de los diferentes especímenes. Inicialmente, las cinéticas de secado tienen pendientes negativas, posteriormente, tienen pendientes cercanas a cero, previendo que se ha alcanzado el estado pendular, donde el agua ligada es la mínima, es decir, el agua que no se puede secar, debido a las propiedades intrínsecas de los especímenes de tapia pisada; por tanto, es este momento en el que los especímenes habían terminado su tiempo de fraguado, aproximadamente a los 15 días.

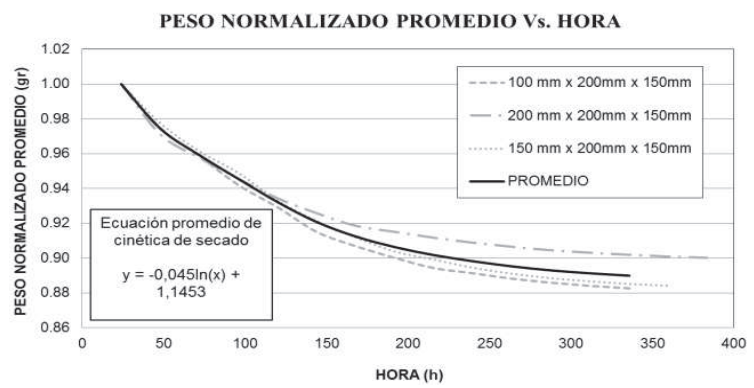

Fig. 12. Peso normalizado promedio vs. Hora para diferentes especímenes [6]

La ecuación promedio aproximada del peso de un muro de tapia pisada está en función del tiempo, y podrá determinar el peso aproximado de cualquier muro a un determinado tiempo si se multiplica el valor obtenido en la ecuación por el peso inicial del muro. Los especímenes no mantuvieron similares resistencias a la compresión promedio debido a que la fuerza de apisonamiento fue variable para cada tamaño de ellos; además, los espesores de las capas de apisonado fueron menores a los utilizados en los especímenes de 500x250x500 mm, aumentando la densidad del material y disminuyendo la relación de vacíos (véase Fig. 13). 


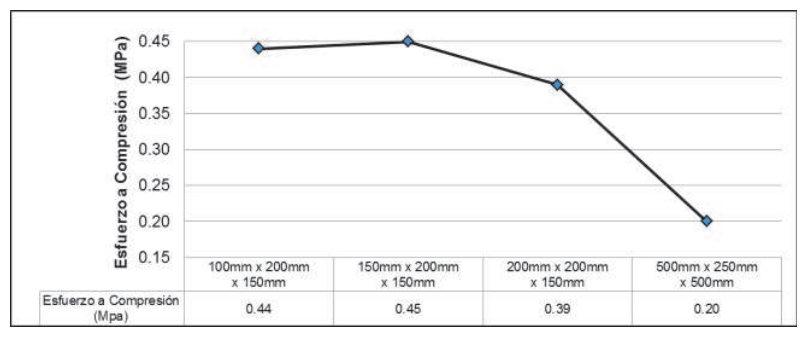

Fig. 13. Resistencia a compresión vs. Tamaño del especímenes [6]

\section{DiscuSIóN}

El material utilizado en la construcción de la tapia pisada depende del sitio donde se va a realizar la construcción; estudios realizados por otras universidades indican material compuesto principalmente por arcillas de baja plasticidad, limos de baja plasticidad y arenas limo-arcillosas (ver Tabla 4).

TABLA 4

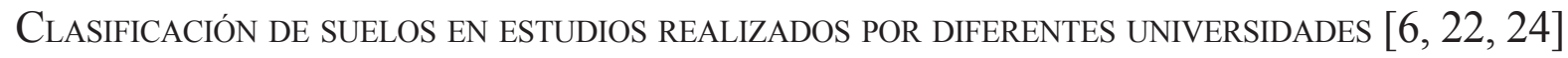

\begin{tabular}{|c|l|c|c|}
\hline Universidad & \multicolumn{1}{|c|}{ Parámetros } & \multicolumn{2}{c|}{ Clasificación } \\
\hline \multirow{4}{*}{ U.F.P.S.O. $(*)$} & El llano & SC-SM & Arena Limo - Arcillosa \\
\cline { 2 - 4 } & La piñuela & SC & Arena Arcillosa \\
\cline { 2 - 4 } & El ramal & CL & Arcilla de Baja Plasticidad \\
\cline { 2 - 4 } & La costas & SM-SC & Arena Limo - Arcillosa \\
\cline { 2 - 4 } & Muestra de Trabajo & SM-SC & Arena Limo - Arcillosa \\
\hline \multirow{2}{*}{$\begin{array}{c}\text { U.F.P.S. } \\
(* *)\end{array}$} & Muestra de Trabajo & - & \\
\hline \multirow{3}{*}{$\begin{array}{c}\text { ANDES } \\
(* * *)\end{array}$} & Tapia Clínica Sta. Bárbara & CL & Arcilla de Baja Plasticidad \\
\cline { 2 - 4 } & Tapia casa Fernández & CL & Arcilla de Baja Plasticidad \\
\cline { 2 - 4 } & Tapia casa Venados & CL & Arcilla de Baja Plasticidad \\
\hline \multirow{2}{*}{$\begin{array}{c}\text { U.I.S. } \\
(* * * *)\end{array}$} & Barichara & ML & Limo de Baja Plasticidad \\
\cline { 2 - 4 } & San Gil & ML & Limo de Baja Plasticidad \\
\cline { 2 - 4 } & Piedecuesta & SM & Arena Limo \\
\hline
\end{tabular}

Existe una relación directa entre el esfuerzo a compresión y el módulo de elasticidad; para especímenes de la fuente de trabajo realizados en Ocaña, Norte de Santander se relacionan matemáticamente (véase la Fig. 14) con un coeficiente de correlación $\mathrm{R}$ de 0.675 . Esta relación directa indica que a mayor esfuerzo a compresión (ftp), mayor será el módulo de elasticidad (E) (ver Fig. 14); además, indica una importante diferencia de valores que dependen de la compactación y del tipo de material.

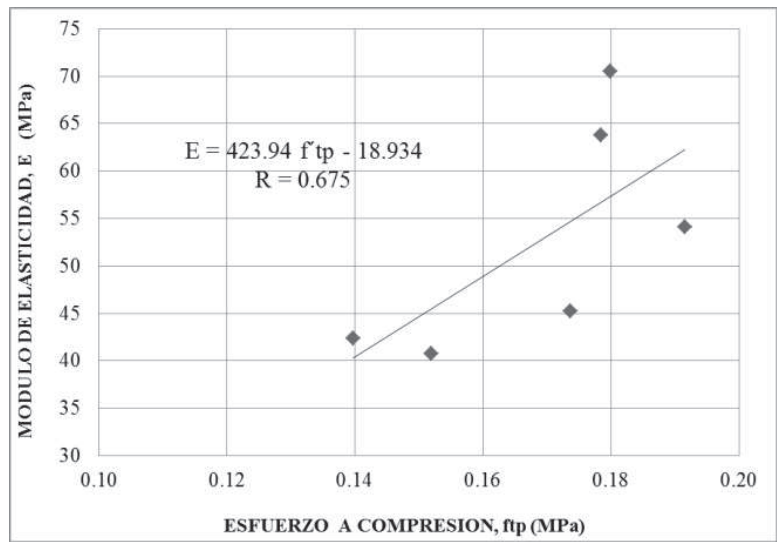

Fig. 14. Relación Esfuerzo a compresión y Módulo de elasticidad [6] 
La resistencia a la flexión es poca, pues en la manipulación puede fallar por peso propio si no se toman las medidas correctivas; esto indicaba que los muros son vulnerables a la flexión.

La resistencia a corte obedece a una distribución normal con un coeficiente de variación del 9.9\%, indicando en el ensayo su repetitividad y valores menores a los observados en mampostería simple. El módulo de corte en tapia pisada $(\mathrm{G})$ tiene alguna dispersión en los datos, pero los resultados son muy cercanos a los encontrados en otras universidades.

El análisis de vulnerabilidad al desplazamiento indicó que la construcción es vulnerable sísmicamente con un índice de flexibilidad de 3.1 y 3.6 en la dirección X y Y, respectivamente, lo cual significa que en la dirección $\mathrm{X}$ es 3.1 veces más flexible que una edificación nueva y que tiene una rigidez de 0.32 veces la rigidez de una construcción reciente, lo cual no es el mejor escenario para una edificación histórica. Mientras que los índices de sobrerresistencia fueron excedidos en diferentes acciones, en tensión y corte no fue evaluado, pues su resistencia es nula; por lo tanto, los muros requieren proveer de resistencia adicional a compresión, flexión, corte y tensión, a fin de tener una construcción sismorresistente.

El comportamiento de la tapia debido a la pérdida de humedad en el tiempo es de orden logarítmico, y para todos los tamaños realizados tiene un comportamiento similar. La pérdida de humedad depende del área de secado y puede variar desde los 14 a 16 días, con porcentajes de agua remanente del $1.57 \%$ al $3.32 \%$ para los especímenes de 100×200x150 mma 150×200x150 $\mathrm{mm}$, respectivamente (ver Fig. 15).

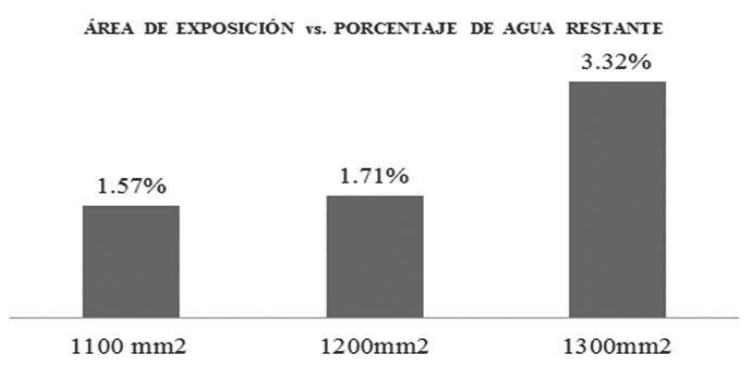

Fig. 15. Área de exposición vs. Porcentaje de agua restante

Los esfuerzos a compresión dependen de la densidad del material (contenido óptimo de humedad) y de la relación de vacíos. En la construcción de los especímenes no fue posible estandarizar la elaboración de las diferentes dimensiones de los especímenes, pues es un proceso de construcción artesanal que presenta alguna dispersión en el esfuerzo a la compresión.

\section{Conclusiones}

El Complejo Histórico de la Gran Convención, patrimonio histórico y cultural de la nación, no es vulnerable por acciones gravitacionales, pero sí lo es sísmicamente, según los resultados de esta investigación; es por ello que la normativa de construcción vigente para Colombia (NSR-10) no considera los muros en tapia pisada elementos de resistencia sísmica.

En la tapia pisada, por ser un elemento construido principalmente por tierra apisonada y algunos elementos como paja y madera, se observó un comportamiento a las acciones de compresión y tracción diagonal tal que no realiza incursiones en el rango no lineal, por lo tanto, su comportamiento es lineal y elástico. 
Por ser un material realizado por artesanos locales, es importante garantizar una densidad del material tal que permita esfuerzos importantes en compresión y tracción diagonal, entre otros; por lo tanto, se sugiere realizar el apisonado con equipos mecánicos que permitan estandarizar la altura de golpeado y el tiempo de compactación y utilizar capas de compactación no mayores a 120 $\mathrm{mm}$, como también garantizar un contenido de humedad óptimo.

El tiempo de fraguado depende del área de exposición y del material; de los resultados de la cinética de secado se puede interpretar que el tiempo de fraguado en los especímenes es de 16 días para material areno-arcilloso, normalmente utilizado en la tapia pisada.

\section{REFERENCIAS}

[1] T. Hodsdon. "Innovations in rammed earth construction". Western Australia Structural Engineer 84, No. 10, p. 30-36. 2006.

[2] D. Easton. The rammed earth house, Rev. edn. White River Junction, VT: Chelsea Green Publishing, 2007.

[3] M. Phillips. "A rammed earth House in Massachusetts". Bulletin of the Association for Preservation Technology Vol. XV, No. 2, p. 33-37, 1983.

[4] P. Walter, R. Keable, J. Martin V. Maniatidis (2005). Rammed earth, design and construction guidelines. BRE. Watford.

[5] Oficina de Planeación Municipal de Ocaña. Plan Básico de Ordenamiento Territorial Municipio de Ocaña 2002-2011, Ocaña, Colombia, 2012.

[6] G. N. Afanador, D. M. Carrascal y CH. M. Bayona. Proyecto de Investigación "Estudio de las propiedades físico-mecánicas de la tapia pisada: Estudio de caso Complejo Histórico de la Gran Convención". Universidad Francisco de Paula Santander, Ocaña, Colombia, 2012.

[7] A. Lee. "El convento franciscano de Ocaña: datos para su historia". Revista Hacaritama, 274, 18-19, 1998.

[8] Instituto Colombiano de Normas Técnicas (NTC). Métodos de ensayo para determinar la resistencia a la compresión de muretes de mampostería, NTC 3495. Bogotá D.C., 2003.

[9] Instituto Colombiano de Normas Técnicas (NTC). Método de ensayo para determinar el módulo de elasticidad estático y la relación de Poisson en concreto a compresión, NTC 4025. Bogotá D.C., 2006.

[10] Instituto Colombiano de Normas Técnicas (NTC). Métodos para muestreo y ensayos de unidad de mampostería y otros productos de arcilla, NTC 4017. Bogotá D.C., 2005.

[11] American Society for Testing and Materials (ASTM). Standard test method for diagonal tension (shear) in masonry assemblages ASTM E519-07. United States, 2007.

[12] Instituto Colombiano de Normas Técnicas (NTC). Suelos. Ensayo para determinar la granulometría por tamizado, NTC 1522. Bogotá D.C., 1.979.

[13] Instituto Nacional de Vías (INVIAS). Análisis granulométrico por medio del hidrómetro (INVE 124-07). Bogotá D.C., 1979a.

[14] Instituto Colombiano de Normas Técnicas (NTC). Suelos. Ensayo para determinar el límite plástico e índice de plasticidad (NTC 1493). 1ra ratificación. Bogotá D.C., $2006 a$. 
[15] Instituto Colombiano de Normas Técnicas (NTC). Suelos. Ensayo para determinar el límite líquido (NTC 1494). 1ra ratificación. Bogotá D.C., 2006b.

[16] Instituto Nacional de Vías (INVIAS). Relaciones de humedad-Masa Unitaria seca en los suelos (INVE 142-07). Bogotá D.C., $1.979 b$.

[17] F. C. Angarita. "A propósito de un cincuentenario: El templo de San Francisco". Revista Hacaritama, Ocaña, 1952.

[18] V. O. García. "La convención de Ocaña: 180 años, Templo de San Francisco: ¿Por qué se escogió a Ocaña como sede de la Convención?". Revista Hacaritama, Ocaña, 1973.

[19] Asociación de Colombiana de Ingeniería Sísmica. Norma Colombiana de Diseño y Construcción Sismo Resistente (NSR-98). Bogotá D.C., 1998.

[20] Asociación Colombiana de Ingeniería Sísmica. Norma sismo resistente 2010. Reglamento colombiano de construcción sismo resistente (NSR-10). Bogotá D.C., 2010 .

[21] SAP2000 V14. Advanced 14.2.0. Structural Analysis Program, computer and structures Inc. Berkely, California, USA, 2010.

[22] R. L. Benítez y C. C. Navas, Influencia de las propiedades físico mecánicas en el material tierra para construcción con tapia pisada. Trabajo de grado Ingeniero Civil. Universidad Industrial de Santander. Facultad de Ingenierías Físico-Mecánicas. Bucaramanga, Colombia, 2009.

[23] M. López. Alternativas de rehabilitación sísmica para edificaciones en adobe y tapia pisada en el barrio La Candelaria de Bogotá. Trabajo de grado no publicado. Bogotá. Universidad de los Andes, 2004.

[24] L. Romero y G. Vergel. Comportamiento mecánico de muretes de bareque compuestos por esqueleto de guadua embebido en matriz de arcilla tratada y elaborados con materiales típicos de la ciudad de San José de Cúcuta. Trabajo de grado no publicado, San José de Cúcuta. Univ. Francisco de Paula Santander. 2006. 